\title{
Blowing up solutions of the modified Novikov-Veselov equation and minimal surfaces
}

\author{
Iskander A. TAIMANOV *
}

\section{Introduction}

In the present article we construct a solution to the modified Novikov-Veselov equation (the two-dimensional generalization of the modified Korteweg-de Vries equation) which has a singularity exactly at one point (Theorem 2).

The solution is given by an explicit formula

$$
\begin{gathered}
\widetilde{U}(x, y, t)=-\frac{3\left(\left(x^{2}+y^{2}+3\right)\left(x^{2}-y^{2}\right)-6 x(C-t)\right)}{Q(x, y, t)}, \\
Q(x, y, t)=\left(x^{2}+y^{2}\right)^{3}+3\left(x^{4}+y^{4}\right)+18 x^{2} y^{2}+9\left(x^{2}+y^{2}\right)+ \\
+9(C-t)^{2}+\left(6 x^{3}-18 x y^{2}-18 x\right)(C-t),
\end{gathered}
$$

from which it is clear that

- it is infinitely differentiable (and even really-analytical) everywhere outside a single point $x=y=0, t=C=$ const at which it is not defined and has different finite limit values along the rays $x / y=$ const, $t=C$, going into this point;

- its restrictions onto all planes $t=$ const decay as $O\left(1 / r^{2}\right)$, and, in particular, have finite $L_{2}$-norms;

*Sobolev Institute of Mathematics, Academician Koptyug avenue 4, 630090, Novosibirsk, Russia, and Department of Mathematics and Mechanics, Novosibirsk State University, Pirogov street 2, 630090 Novosibirsk, Russia; e-mail: taimanov@math.nsc.ru. The work was supported by RSF (grant 14-11-00441). 
- the first integral (conservation law) $\int_{\mathbb{R}^{2}} \widetilde{U}^{2} d x d y$ has the same value equal to $3 \pi$ for all times $t \neq C$ and jumps to $2 \pi$ for $t=C$.

The method of constructing such solutions is given by Theorem 1 and we consider in detail only only simplest example. It is based on the geometrical interpretation [1] of the Moutard transformation for two-dimensional Dirac operators [2].

\section{Preliminary facts}

\subsection{The modified Novikov-Veselov equation}

The modified Novikov-Veselov (mNV) equation has the form

$$
U_{t}=\left(U_{z z z}+3 U_{z} V+\frac{3}{2} U V_{z}\right)+\left(U_{\bar{z} \bar{z} \bar{z}}+3 U_{\bar{z}} \bar{V}+\frac{3}{2} U \bar{V}_{\bar{z}}\right)
$$

where

$$
V_{\bar{z}}=\left(U^{2}\right)_{z},
$$

$z=x+i y \in \mathbb{C}, U$ is a real-valued function.

For making the equation correctly-posed we have to uniquely resolve the constraint which defines $V$. For instance, for fast decaying solutions $U$ we may do that by assuming that $V$ is also fast decaying.

This equation takes the form of Manakov's $L, A, B$-triple:

$$
\mathcal{D}_{t}+[\mathcal{D}, \mathcal{A}]-\mathcal{B D}=0
$$

where $\mathcal{D}$ is a two-dimensional Dirac operator:

$$
\mathcal{D}=\left(\begin{array}{cc}
0 & \partial \\
-\bar{\partial} & 0
\end{array}\right)+\left(\begin{array}{cc}
U & 0 \\
0 & U
\end{array}\right)
$$

$\partial=\frac{\partial}{\partial z}$ and $\bar{\partial}=\frac{\partial}{\partial \bar{z}}$

$$
\begin{gathered}
\mathcal{A}=\partial^{3}+\bar{\partial}^{3}+ \\
+3\left(\begin{array}{cc}
V & 0 \\
U_{z} & 0
\end{array}\right) \partial+3\left(\begin{array}{cc}
0 & -U_{\bar{z}} \\
0 & \bar{V}
\end{array}\right) \bar{\partial}+\frac{3}{2}\left(\begin{array}{cc}
V_{z} & 2 U \bar{V} \\
-2 U V & \bar{V}_{\bar{z}}
\end{array}\right), \\
\mathcal{B}=3\left(\begin{array}{cc}
-V & 0 \\
-2 U_{z} & V
\end{array}\right) \partial+3\left(\begin{array}{cc}
\bar{V} & 2 U_{\bar{z}} \\
0 & -\bar{V}
\end{array}\right) \bar{\partial}+\frac{3}{2}\left(\begin{array}{cc}
\bar{V}_{\bar{z}}-V_{z} & 2 U_{\bar{z} \bar{z}} \\
-2 U_{z z} & V_{z}-\bar{V}_{\bar{z}}
\end{array}\right)
\end{gathered}
$$


If $U$ depends only on $x$ and therewith $V=U^{2}$, then this equation reduces to the modified Korteweg-de Vries equation

$$
U_{t}=\frac{1}{4} U_{x x x}+6 U_{x} U^{2}
$$

The mNV equation was introduced in [3] and its name is due to the Novikov-Veselov equation introduced in [4, 5]) which is a similar 2-dimensional generalization of the Korteweg-de Vries equation.

\subsection{The Weierstrass representation of minimal surfaces}

A surface in $\mathbb{R}^{3}$ is called minimal if its mean curvature vanishes everywhere:

$$
H=0 \text {. }
$$

The Weierstrass representation corresponds to every pair of holomorphic functions

$$
\psi_{1}, \bar{\psi}_{2}: \mathcal{U} \rightarrow \mathbb{C}
$$

a minimal surface

$$
F: \mathcal{U} \rightarrow \mathbb{R}^{3}, \quad F=\left(u^{1}, u^{2}, u^{3}\right)
$$

given by the formulas

$$
\begin{gathered}
u^{1}(P)=\frac{i}{2} \int_{P_{0}}^{P}\left(\left(\psi_{1}^{2}+\bar{\psi}_{2}^{2}\right) d z-\left(\bar{\psi}_{1}^{2}+\psi_{2}^{2}\right) d \bar{z}\right)+u^{1}\left(P_{0}\right), \\
u^{2}(P)=\frac{1}{2} \int_{P_{0}}^{P}\left(\left(\bar{\psi}_{2}^{2}-\psi_{1}^{2}\right) d z+\left(\psi_{2}^{2}-\bar{\psi}_{1}^{2}\right) d \bar{z}\right)+u^{2}\left(P_{0}\right), \\
u^{3}(P)=\int_{P_{0}}^{P}\left(\psi_{1} \bar{\psi}_{2} d z+\bar{\psi}_{1} \psi_{2} \bar{z}\right)+u^{3}\left(P_{0}\right),
\end{gathered}
$$

where $\left(u^{1}, u^{2}, u^{3}\right)$ are the Euclidean coordinates in $\mathbb{R}^{3}, P_{0} \in \mathcal{U}$ and the integral is taken over a path in $\mathcal{U}$ joining $P_{0}$ and $P$. If $\mathcal{U}$ is simply-connected, then the integral does not depend on a choice of a path. This is an immersion outside branch points where the induced metric

$$
d s^{2}=e^{2 \alpha} d z d \bar{z}=\left(\left|\psi_{1}\right|^{2}+\left|\psi_{2}\right|^{2}\right)^{2} d z d \bar{z}
$$


vanishes. The unit normal vector is equal to

$$
\mathbf{n}=\frac{1}{\left|\psi_{1}\right|^{2}+\left|\psi_{2}\right|^{2}}\left(i\left(\psi_{1} \psi_{2}-\bar{\psi}_{1} \bar{\psi}_{2}\right),-\left(\psi_{1} \psi_{2}+\bar{\psi}_{1} \bar{\psi}_{2}\right),\left(\left|\psi_{2}\right|^{2}-\left|\psi_{1}\right|^{2}\right)\right)
$$

The formulas (5) define a surface up to translations, i.e. up to $F\left(P_{0}\right)$.

It is well-known that every minimal surface in $\mathbb{R}^{3}$ admits such a representation.

\subsection{The Enneper surface}

The Enneper surface is an immersed (not embedded) minimal surface defined via the formulas (15) by

$$
\psi_{1}=z, \quad \psi_{2}=1 \text {. }
$$

Substituting that into (5), we obtain

$$
\begin{gathered}
u^{1}(x, y)=y\left(\frac{y^{2}}{3}-x^{2}-1\right)+u_{0}^{1}, \\
u^{2}(x, y)=x\left(1+y^{2}-\frac{x^{2}}{3}\right)+u_{0}^{2}, \\
u^{3}(x, y)=x^{2}-y^{2}+u_{0}^{3},
\end{gathered}
$$

where $\mathbf{u}_{0}=\left(u_{0}^{1}, u_{0}^{2}, u_{0}^{3}\right)$ is the image of the origin $x=y=0$ under an immersion.

\section{The Moutard transformation}

Let

$$
\psi=\left(\begin{array}{l}
\psi_{1} \\
\psi_{2}
\end{array}\right)
$$

be a solution of the Dirac equation

$$
\mathcal{D} \psi=0
$$

where $\mathcal{D}$ is the Dirac operator (3). It is clear that

$$
\psi^{*}=\left(\begin{array}{c}
-\bar{\psi}_{2} \\
\bar{\psi}_{1}
\end{array}\right)
$$


satisfies the same equation. Let us form a matrix-valued function $\Psi$ from $\psi$ and $\psi^{*}$ as follows

$$
\Psi=\left(\begin{array}{cc}
\psi_{1} & -\bar{\psi}_{2} \\
\psi_{2} & \bar{\psi}_{1}
\end{array}\right) .
$$

It meets the matrix Dirac equation

$$
\mathcal{D} \Psi=0 .
$$

We denote by $H$ a space formed by all matrices of the form

$$
\left(\begin{array}{cc}
\alpha & \beta \\
-\bar{\beta} & \bar{\alpha}
\end{array}\right), \quad \alpha, \beta \in \mathbb{C},
$$

and put

$$
\Gamma=\left(\begin{array}{cc}
0 & 1 \\
-1 & 0
\end{array}\right) .
$$

It is evident that $H$ is closed under products and $\Gamma, \Psi \in H$.

For $U=0$ we have the operator

$$
\mathcal{D}_{0}=\left(\begin{array}{cc}
0 & \partial \\
-\bar{\partial} & 0
\end{array}\right)
$$

and vector functions $\psi$ which define minimal surfaces via (5) are exactly solutions of $\mathcal{D}_{0} \psi=0$.

Given scalar functions $U$ and $V$, let us correspond to $H$-valued functions $\Phi$ and $\Psi$ a matrix-valued 1 -form

$$
\begin{gathered}
\widetilde{\omega}(\Phi, \Psi)=\Phi^{\top} \Psi d y-i \Phi^{\top} \sigma_{3} \Psi d x+\left[i\left(\Phi_{y y}^{\top} \sigma_{3} \Psi+\Phi^{\top} \sigma_{3} \Psi_{y y}-\Phi_{y}^{\top} \sigma_{3} \Psi_{y}\right)+\right. \\
\left.2 i U\left(\Phi_{y}^{\top} \sigma_{2} \Psi-\Phi^{\top} \sigma_{2} \Psi_{y}\right)+\Phi^{\top}\left(\begin{array}{cc}
i U^{2}-3 i V & -i U_{x} \\
-i U_{x} & -i U^{2}+3 i \bar{V}
\end{array}\right) \Psi\right] d t= \\
-\frac{i}{2}\left(\Phi^{\top} \sigma_{3} \Psi+\Phi^{\top} \Psi\right) d z-\frac{i}{2}\left(\Phi^{\top} \sigma_{3} \Psi-\Phi^{\top} \Psi\right) d \bar{z}+ \\
{\left[-i\left(\left(\Phi_{z z}^{\top}+\Phi_{\bar{z} \bar{z}}^{\top}-2 \Phi_{z \bar{z}}^{\top}\right) \sigma_{3} \Psi+\Phi^{\top} \sigma_{3}\left(\Psi_{z z}+\Psi_{\bar{z} \bar{z}}-2 \Psi_{z \bar{z}}\right)-\right.\right.} \\
\left.\left(\Phi_{z}^{\top}-\Phi_{\bar{z}}^{\top}\right) \sigma_{3}\left(\Psi_{z}-\Psi_{\bar{z}}\right)\right)-2 U\left(\left(\Phi_{z}^{\top}-\Phi_{\bar{z}}^{\top}\right) \sigma_{2} \Psi-\Phi^{\top} \sigma_{2}\left(\Psi_{z}-\Psi_{\bar{z}}\right)\right)+ \\
\left.\Phi^{\top}\left(\begin{array}{cc}
i U^{2}-3 i V & -i\left(U_{z}+U_{\bar{z}}\right) \\
-i\left(U_{z}+U_{\bar{z}}\right) & -i U^{2}+3 i \bar{V}
\end{array}\right) \Psi\right] d t
\end{gathered}
$$


and matrix-valued functions

$$
\begin{gathered}
\widetilde{S}(\Phi, \Psi)(z, \bar{z}, t)=\Gamma \int_{0}^{z} \widetilde{\omega}(\Phi, \Psi), \\
K(\Psi)=\Psi \widetilde{S}^{-1}(\Psi, \Psi) \Gamma \Psi^{\top} \Gamma^{-1}, \\
M(\Psi)=\Gamma \Psi_{y} \Psi^{-1} \Gamma^{-1}=i \Gamma\left(\Psi_{z}-\Psi_{\bar{z}}\right) \Psi^{-1} \Gamma^{-1} .
\end{gathered}
$$

The following Moutard transformation of solutions to the mNV equation was introduced in [2].

Proposition 1 ([2]) Let $U(z, \bar{z}, t)$ and $V(z, \bar{z}, t)$ satisfy the $m N V$ equation (2), $\mathcal{D}$ is the family of Dirac operators with potentials $U(z, \bar{z}, t)$, and $\Psi_{0}(z, \bar{z}, t)$ satisfy the system

$$
\mathcal{D} \Psi_{0}=0, \quad \frac{\partial \Psi_{0}}{\partial t}=\mathcal{A} \Psi_{0}
$$

where $\mathcal{A}$ has the form (4). Then

1. the matrices $K\left(\Psi_{0}\right)$ and $M\left(\Psi_{0}\right)$ take the form

$$
K=\left(\begin{array}{cc}
i W & a \\
-\bar{a} & -i W
\end{array}\right), \quad M=\left(\begin{array}{cc}
b & c \\
-\bar{c} & \bar{b}
\end{array}\right),
$$

with $W$ real valued;

2. for every solution $\Psi$ of the equations (8) and

$$
\frac{\partial \Psi}{\partial t}=\mathcal{A} \Psi
$$

the function $\widetilde{\Psi}$ of the form

$$
\widetilde{\Psi}=\Psi-\Psi_{0} \widetilde{S}^{-1}\left(\Psi_{0}, \Psi_{0}\right) \widetilde{S}\left(\Psi_{0}, \Psi\right)
$$

satisfies the equations

$$
\widetilde{\mathcal{D}} \widetilde{\Psi}=0
$$

for the Dirac operator $\widetilde{\mathcal{D}}$ with potential

$$
\widetilde{U}=U+W
$$


and

$$
\frac{\partial \widetilde{\Psi}}{\partial t}=\widetilde{\mathcal{A}} \widetilde{\Psi}
$$

where $\widetilde{\mathcal{A}}$ takes the form (4) with $U$ replaced by $\widetilde{U}$ and $V$ replaced by $\widetilde{V}$ :

$$
\widetilde{V}=V+2 U W+a^{2}+2(a \bar{b}-i \bar{c} W)
$$

3. the function $\widetilde{U}$ is real-valued and $\widetilde{U}$ and $\widetilde{V}$ satisfy the $m N V$ equation

$$
\begin{gathered}
\widetilde{U}_{t}=\left(\widetilde{U}_{z z z}+3 \widetilde{U}_{z} \widetilde{V}+\frac{3}{2} \widetilde{U}_{\bar{V}}\right)+\left(\widetilde{U}_{\bar{z} \bar{z} \bar{z}}+3 \widetilde{U}_{\bar{z}} \overline{\widetilde{V}}+\frac{3}{2} \widetilde{U}_{\bar{V}} \overline{\tilde{V}}_{\bar{z}}\right), \\
\widetilde{V}_{\bar{z}}=\left(\widetilde{U}^{2}\right)_{z}
\end{gathered}
$$

\section{Minimal surfaces and blowing up solutions of the $\mathrm{mNV}$ equation}

Let us apply Proposition 1 to the operator with $U=0$. Although this is a stationary solution of the $\mathrm{mNV}$ equation, the Moutard transformation leads to a non-trivial non-stationary solution of the mNV equation. A similar effect was found and used for the Novikov-Veselov equation [6, 7].

By straightforward computations we derive

Theorem 1 Let $\psi_{1}(z, \bar{z}, t)$ and $\psi_{2}(z, \bar{z}, t)$ be a functions which satisfy the equations

$$
\begin{gathered}
\bar{\partial} \psi_{1}=\bar{\partial} \bar{\psi}_{2}=0, \\
\frac{\partial \psi_{1}}{\partial t}=\frac{\partial^{3} \psi_{1}}{\partial z^{3}}, \quad \frac{\partial \psi_{2}}{\partial t}=\frac{\partial^{3} \psi_{2}}{\partial \bar{z}^{3}} .
\end{gathered}
$$

Then

$$
\widetilde{S}\left(\Psi_{0}, \Psi_{0}\right)(z, \bar{z}, t)=\left(\begin{array}{cc}
i u^{3} & -u^{1}-i u^{2} \\
u^{1}-i u^{2} & -i u^{3}
\end{array}\right)+i \int_{0}^{t}\left(\begin{array}{cc}
w & \bar{v} \\
v & -w
\end{array}\right) d \tau,
$$

where

$$
\Psi_{0}=\left(\begin{array}{cc}
\psi_{1} & -\bar{\psi}_{2} \\
\psi_{2} & \bar{\psi}_{1}
\end{array}\right)
$$


the minimal surfaces $F(z, \bar{z}, t)=\left(u^{1}, u^{2}, u^{3}\right)$ are defined by $\psi_{1}$ and $\psi_{2}$ by (5) with $\mathbf{u}_{0}$ independent on $t$ and

$$
\begin{gathered}
v=\left(\psi_{1, z}^{2}-\psi_{2, \bar{z}}^{2}\right)-2\left(\psi_{1} \psi_{1, z z}-\psi_{2} \psi_{2, \bar{z} \bar{z}}\right) \\
w=\psi_{1, z} \bar{\psi}_{2, z}+\bar{\psi}_{1, \bar{z}} \psi_{2, \bar{z}}-\psi_{1, z z} \bar{\psi}_{2}-\psi_{1} \bar{\psi}_{2, z z}-\bar{\psi}_{1, \bar{z} \bar{z}} \psi_{2}-\bar{\psi}_{1} \psi_{2, \bar{z} \bar{z}}
\end{gathered}
$$

Theorem 1 shows that $\widetilde{S}$ is a deformed minimal surface which depends on $t$ and is given by the second term in (12). We come to the following conclusion

- to obtain a blowing up solution of the $m N V$ equation we have to find a pair of $\psi_{1}$ and $\psi_{2}$ which satisfy the conditions of Theorem 1 and such that the matrix $\widetilde{S}$ degenerates at some moment of time.

The simplest candidate is given by the Enneper surface. In this case

$$
\psi_{1}=z, \quad \psi_{2}=1, \quad v=1, \quad w=0 .
$$

We put the image of the origin to be

$$
u_{0}^{1}=u_{0}^{3}=0, \quad u_{0}^{2}=C>0,
$$

and, by (7), compute

$$
\widetilde{S}(x, y, t)=\left(\begin{array}{cc}
i u^{3} & -u^{1}-i u^{2}+i t \\
u^{1}-i u^{2}+i t & -i u^{3}
\end{array}\right)=\left(\begin{array}{cc}
\gamma & \delta \\
-\bar{\delta} & \bar{\gamma}
\end{array}\right)
$$

with

$$
\gamma=i\left(x^{2}-y^{2}\right), \quad \delta=-y\left(\frac{y^{2}}{3}-x^{2}-1\right)-i\left[x\left(1+y^{2}-\frac{x^{2}}{3}\right)+C-t\right] .
$$

We also easily derive that

$$
\begin{gathered}
K=\left(\begin{array}{cc}
z & -1 \\
1 & \bar{z}
\end{array}\right) \widetilde{S}^{-1}\left(\begin{array}{cc}
\bar{z} & 1 \\
-1 & z
\end{array}\right), \\
M=\frac{i}{1+|z|^{2}}\left(\begin{array}{cc}
-z & -1 \\
-1 & \bar{z}
\end{array}\right),
\end{gathered}
$$


and therefore

$$
\begin{gathered}
W=-i \frac{|z|^{2} \bar{\gamma}+\gamma+\delta z-\bar{\delta} \bar{z}}{|\gamma|^{2}+|\delta|^{2}}, \quad a=\frac{z(\bar{\gamma}-\gamma)-\delta z^{2}-\bar{\delta}}{|\gamma|^{2}+|\delta|^{2}}, \\
b=-\frac{i z}{1+|z|^{2}}, \quad c=-\frac{i}{1+|z|^{2}} .
\end{gathered}
$$

Since $U=V=0$, we finally obtain

$$
\begin{gathered}
\tilde{U}=-i \frac{|z|^{2} \bar{\gamma}+\gamma+\delta z-\bar{\delta} \bar{z}}{|\gamma|^{2}+|\delta|^{2}} \\
\widetilde{V}=\frac{\left(z(\bar{\gamma}-\gamma)-\delta z^{2}-\bar{\delta}\right)^{2}}{\left(|\gamma|^{2}+|\delta|^{2}\right)^{2}}+\frac{2 \widetilde{U}}{1+|z|^{2}}-2 \frac{i z\left(z(\bar{\gamma}-\gamma)-\delta z^{2}-\bar{\delta}\right)}{\left(|\gamma|^{2}+|\delta|^{2}\right)\left(1+|z|^{2}\right)}
\end{gathered}
$$

where $\gamma$ and $\delta$ are given by (13).

Put $r=\sqrt{x^{2}+y^{2}}=|z|$. It is clear that

$$
b=O\left(\frac{1}{r}\right), \quad c=O\left(\frac{1}{r^{2}}\right) \quad \text { as } r \rightarrow \infty .
$$

By (13), we have

$$
\gamma=O\left(r^{2}\right), \quad \delta=O\left(r^{3}\right), \quad a=O\left(\frac{1}{r}\right)
$$

and finally we derive that

$$
\widetilde{U}=O\left(\frac{1}{r^{2}}\right), \quad \widetilde{V}=O\left(\frac{1}{r^{2}}\right) \quad \text { as } r \rightarrow \infty .
$$

These functions $\widetilde{U}$ and $\widetilde{V}$ may have singularities only at points where $|\gamma|^{2}+|\delta|^{2}=0$, i.e. exactly at the points where the moving Enneper surface $\left(u^{1}, u^{2}-t, u^{3}\right)$ hits the origin. This motion preserves $u^{1}$ and $u^{3}$ and, since we assume that $u_{0}^{1}=u_{0}^{3}=0$, it is clear from (17) that $u^{1}=u^{3}=0$ if and only if $x=y=0$. However at $x=y=0$ we have $u^{2}=C=$ const and hence

$$
|\gamma|^{2}+|\delta|^{2}=0 \text { if and only if } \quad t=C .
$$

Theorem 2 The functions $\widetilde{U}(14)$ and $\widetilde{V}$ (15) with $\gamma$ and $\delta$ given by (13) 
1. satisfy the modified Novikov-Veselov equation (11);

2. decay at least quadratically in $r: \widetilde{U}=O\left(r^{-2}\right), \widetilde{V}=O\left(r^{-2}\right)$;

3. are really analytical $t \neq C$;

4. have singularities exactly at $x=y=0, t=C$. At this point $\widetilde{U}$ is not defined and

$$
\lim _{r \rightarrow 0, \varphi=\mathrm{const}} \tilde{U}(z, \bar{z}, C)=-\cos 2 \varphi \quad \text { for } z=r e^{i \varphi}
$$

5.

$$
\int_{\mathbb{R}^{2}}|\widetilde{U}|^{2} d x d y= \begin{cases}3 \pi & \text { for } t \neq C, \\ 2 \pi & \text { for } t=C .\end{cases}
$$

The statements 1-3 of Theorem are established above.

The statement 4 follows from the formula (1) which is straightforwardly derived from (14). For $C=t$ this formula reduces to

$$
\widetilde{U}=-\frac{3 r^{2}\left(r^{2}+3\right) \cos 2 \varphi}{r^{2}\left(r^{4}+3 r^{2}\left(1+\sin ^{2} 2 \varphi\right)+9\right)} .
$$

Here $r$ and $\varphi$ are the polar coordinates: $x+i y=z e^{i \varphi}=r(\cos \varphi+i \sin \varphi)$.

The statement 5 will be proved in the next section.

\section{Geometry of the blowing up solution of the mNV equation}

The exposition of the geometrical properties of the solution is based on the explicit formulas for the action of the Möbius inversion on the Weierstrass representation data [1] and on the relation of $\int U^{2} d x d y$ to the Willmore functional [8].

Any surface in $\mathbb{R}^{3}$ is defined by the formulas (5) (the Weierstrass representation) where a spinor $\psi$ satisfies the Dirac equation

$$
\mathcal{D} \psi=\left[\left(\begin{array}{cc}
0 & \partial \\
-\bar{\partial} & 0
\end{array}\right)+\left(\begin{array}{cc}
U & 0 \\
0 & U
\end{array}\right)\right] \psi
$$


defines a surface in $\mathbb{R}^{3}$ via formulas (5). Therewith $z$ is a conformal parameter on surface such that the induced metric takes the form (마) and the real-valued potential $U$ is equal to

$$
U=\frac{e^{\alpha} H}{2}=\frac{\left(\left|\psi_{1}\right|^{2}+|\psi|^{2}\right) H}{2}
$$

with $H$ the mean curvature of the surface [9, 8, 10]. For $H=0$, i.e. for minimal surfaces, this representation is use the Weierstrass representation of minimal surfaces exposed above.

In [1] we show that

1. the reduced matrix function

$$
S\left(\Psi_{0}, \Psi_{0}\right)(x, y, t)=\Gamma \int-\frac{i}{2}\left(\Psi_{0}^{\top}\left(\sigma_{3}+1\right) \Psi_{0} d z+\Psi_{0}^{\top}\left(\sigma_{3}-1\right) \Psi_{0} d \bar{z}\right)
$$

where

$$
\Psi_{0}=\left(\begin{array}{cc}
\psi_{1} & -\bar{\psi}_{2} \\
\psi_{2} & \bar{\psi}_{1}
\end{array}\right)
$$

is equal to

$$
\left(\begin{array}{cc}
i u^{3} & -u^{1}-i u^{2} \\
u^{1}-i u^{2} & -i u^{3}
\end{array}\right) \in s u(2) \approx \mathbb{R}^{3}
$$

where $\Sigma_{t}=\left(u^{1}, u^{2}, u^{3}\right)$ is a surface defined up to translations by $\psi=$ $\left(\begin{array}{l}\psi_{1} \\ \psi_{2}\end{array}\right)$ via (5) at every moment $t$;

2. the Möbius inversion of $\mathbb{R}^{3} \cup\{\infty\}=S^{3}$ in terms of (20) takes a simple form

$$
S \rightarrow S^{-1}
$$

and if $\Psi_{0}$ defines a surface $\Sigma$, then the inverted surface is defined by

$$
\widetilde{\Psi}=\Psi_{0} \cdot S^{-1}
$$

via the same formulas (5);

3. the potential $U$ is transformed by the the inversion by the formula (10), i.e. by the Moutard transformation given in Proposition 1, with $\widetilde{S}$ replaced by $S$ in the definition of $K(\Psi)$. 
The blowing up solution of the mNV equation exposed in Section 4 has a very simple geometrical meaning:

- the stationary function $\psi=\left(\begin{array}{c}z \\ 1\end{array}\right)$ defines an immersion $S_{0}$ of the Enneper surface $\Sigma_{0}$, the matrix function $\widetilde{S}$ is equal to

$$
\widetilde{S}(x, y, t)=S_{0}(x, y)+\left(\begin{array}{cc}
0 & i t \\
i t & 0
\end{array}\right)
$$

and defines a rigid translation $\Sigma_{t}$ of the initial Enneper surface along the $u^{2}$ axis: $u^{2} \longrightarrow u^{2}-t$.

Since all surfaces $\Sigma_{t}$ are minimal, they have the same potential $U=0$, however the potentials of their inversions $\Sigma_{t}^{-1}$ are different and are equal to $\widetilde{U}(x, y, t)$ (14).

The Enneper surfaces $\Sigma_{t}$ hit the origin only at one point $x=y=0$ and only at one moment of time $t=C$ and therewith the inversion maps this point into infinity, $\Sigma_{C}^{-1}$ becomes noncompact, and the potential $\widetilde{U}$ achieves a singularity at $x=y=0, t=C$.

The quantity $4 \int \widetilde{U}^{2} d x d y$ is the conservation law of the mNV equation and is equal to the value of Willmore functional (the integral of the squared mean curvature) at the surface, i.e. in our case at $\Sigma_{t}^{-1}$ [8].

Now the statement 5 of Theorem 2 follows, for instance, from computations of the values of Willmore functional for inverted Enneper surfaces [11.

\section{Final remarks}

1) The deformation $\Sigma_{t}^{-1}$ is an example of the mNV evolution of surfaces introduced in [9] for surfaces "induced" by the formulas (5).

2) The constructed solution is special in many respects:

a) $\widetilde{S}(x, y, t)$ splits into $S_{0}(x, y)+P(t)$, i.e. describes a rigid motion of a minimal surface;

b) the inverted Enneper surfaces have many interesting geometrical features and, in particular, they are branched Willmore spheres [11]. 
Do rigid motions of other minimal surfaces in the same manner correspond to integrable soliton equations?

3) Other computable interesting examples can be found by using the higher order Enneper surfaces defined by the spinors $\psi=\left(\begin{array}{c}z^{k} \\ 1\end{array}\right)$ and soliton spheres (which are not minimal surfaces) [12, 13].

4) The results of this paper were briefly announced in [14].

\section{References}

[1] Taimanov, I.A.: The Moutard transformation of two-dimensional Dirac operators and the Möbius geometry. arXiv:1408.4464.

[2] Delong Yu, Q.P. Liu, and Shikun Wang: Darboux transformation for the modified Veselov-Novikov equation. J. of Physics A 35 (2001), 3779-3785.

[3] Bogdanov, L.V.: The Veselov-Novikov equation as a natural generalization of the Korteweg-de Vries equation. Theoret. and Math. Phys. 70:2 (1987), 219-223.

[4] Veselov, A.P., and Novikov, S.P.: Finite-zone, two-dimensional potential Schrödinger operators. Explicit formulas and evolution equations. Soviet Math. Dokl. 30 (1984), 588-591.

[5] Veselov, A.P., and Novikov, S.P.: Finite-gap two-dimensional Schrödinger operators. Potential operators. Soviet Math. Dokl. 30 (1984), 705-708.

[6] Taimanov, I.A., and Tsarev, S.P.: Two-dimensional rational solitons constructed by means of the Moutard transformations, and their blowup. Theoret. and Math. Phys. 157 (2008), 1525-1541.

[7] Taimanov, I.A., and Tsarev, S.P.: Blowing up solutions of the Veselov-Novikov equation. Dokl. Math. 77:3 (2008), 467-468.

[8] Taimanov, I.A.: Modified Novikov-Veselov equation and differential geometry of surfaces. Amer. Math. Soc. Transl., Ser. 2, V. 179, 1997, pp. 133-151. 
[9] Konopelchenko, B.G.: Induced surfaces and their integrable dynamics. Stud. Appl. Math. 96 (1996), 9-52.

[10] Taimanov, I.A.: The two-dimensional Dirac operator and the theory of surfaces. Russian Math. Surveys 61:1 (2006), 79-159.

[11] Lamm, T., and Huy The Nguyen: Branched Willmore spheres. Journal für die Reine und Angewandte Mathematik (published online May 7, 2013).

[12] Taimanov, I.A.: The Weierstrass representation of spheres in $\mathbb{R}^{3}$, Willmore numbers, and soliton spheres. Proc. Steklov Inst. Math. 225:2 (1999), 322-343.

[13] Bohle, C., and Peters, G.P.: Soliton spheres. Trans. Amer. Math. Soc. 363:10 (2011), 5419-5463.

[14] Taimanov, I.A.: A fast decaying solution to the modified NovikovVeselov equation with a one-point singularity. arXiv:1408.4723. 